\title{
Effect of Pentoxifylline on Recurrence in Patients With Multiple Sclerosis: A Non-Randomized Double-Blinded Placebo Controlled Clinical Trial
}

\author{
Akbar Hamzei Moghaddam,', Rostam Seiffaddini,' Asma Najafzadeh,," Hossien Ali \\ Ghaffaripour, ${ }^{2}$ Peyman Arasteh, ${ }^{3}$ Zeinab Movahhedi, ${ }^{4}$ and Narges Khanjani ${ }^{1}$ \\ ${ }^{1}$ Department of Neurology, Neurology Research Center, Kerman University of Medical Sciences, Kerman, IR Iran \\ ${ }_{2}^{2}$ Pediatric Respiratory Disease Research Center, National Research Institute of Tuberculosis and Lung Disease, Shahid Beheshti University of Medical Sciences, Tehran, IR Iran \\ ${ }^{3}$ Students Research Committee, Shiraz University of Medical Sciences, Shiraz, IR Iran \\ $4^{4} \mathrm{PhD}$ Student of Nursing, Department of Nursing, Kerman University of Medical Sciences, Kerman, IR Iran \\ *Corresponding author:Asma Najafzadeh, Department of Neurology, Neurology Research Center, Kerman University of Medical Sciences, Kerman, IR Iran. E-mail: asma.najafzade@gmail.com \\ Received 2014 April 16; Accepted 2014 June 10.
}

\begin{abstract}
Background: The role of the immune system in multiple sclerosis (MS) has been proved. Pentoxifylline has an inhibitory effect on phosphodiesterase and the cytokine products. In some studies therapeutic effects of pentoxifylline have been documented in MS patients. Objectives: The aim of our study was to investigate the effects of pentoxifylline on the recurrence and the disease course in MS patients. Patients and Methods: In our double-blinded clinical trial study, 44 newly diagnosed relapsing-remitting MS patients were studied for 12 months. They were divided into 2 groups: in group 1, 22 patients received interferon and oral pentoxifylline (800 mg daily for 2 months) and in group 2 (control group), 22 patients received interferon with a placebo. The monthly attack rates and the clinical condition of the patients, using the expanded disability status scale(EDSS), were measured in 3 different times along the study (beginning of the treatment, 6 months after and at the end of the treatment). Analysis of the data was done using t-test and the nonparametric Mann-Whitney U test. Results: There was not any significant difference between the 2 groups in terms of age and sex. The recurrence in the first year was $0.4 \%$ and 0 in group 1 and 2, respectively. Also there was not any difference in the clinical course and the recurrence of the disease between two groups in one year of follow-up.

Conclusions: Adding pentoxifylline to interferon does not have any synergistic therapeutic effects on the reduction of relapse frequency and EDSS in patients with relapsing-remitting multiple sclerosis in comparison with interferon.
\end{abstract}

Keywords: Multiple Sclerosis, Relapse, Pentoxifylline

\section{Background}

Multiple sclerosis (MS) is one of the most common demyelinating diseases of the central nervous system, which also considered the main leading cause of disability in young adults [1]. The symptoms of the disease at early stages result from the axonal damage and conduction block that occurs in demyelinated nerve segments (plaque). The clinical symptoms can range from mild and periodic to severe and persistent or progressive, depending on the location of lesions and their spread [1]. Although MS has four clinical course patterns, a majority of the patients ( $85 \%$ to $90 \%$ ) belongs in relapsing-remitting pattern (RRMS) [2]. The etiology of multiple sclerosis is unknown [1-3].

Clinical manifestation of disease is caused by complex interactions between environmental factors and predisposing genes, whereas an inappropriate immune response might damage myelin sheet, oligodendrocytes, axons, and neurons [1-3]. Multiple sclerosis is probably mediated by the Th1 lymphocytes [4]. The original Th1 cytokines include: IL-2, IFN- $\gamma$ and the tumor necrosis factor $\beta$ (TNF- $\beta$ ) [1] that are myelinotoxic and increases the inflammatory response $[5,6]$. In MS patients, the amount of interleukins, IFN- $\gamma$ and TNF- $\beta$ increase in white blood cells before an attack [7, 8], and hence the cytokines exacerbate disease progression and worse MS symptoms [9-11].

In autoimmune diseases with unknown antigens, such as multiple sclerosis, immune regulatory therapies are developed to change cytokines by inhibiting the production of inflammatory cytokines and inducing protective cytokines similar to Th cells [12]. Immunomodulatory therapies are considered the most responsive treatment in the relapsing-remitting pattern of MS with periodic inflammation [2]. However, all treatments can only prevent the disease attack; there is no cure for MS [13].

Since the maximal efficacy of current drugs to control attack is about 30\% [1], today's efforts focus on finding drugs that are able to decrease the attacks via changes

Copyright (C) 2015, Zahedan University of Medical Sciences. This is an open-access article distributed under the terms of the Creative Commons Attribution-NonCommercial 4.0 International License (http://creativecommons.org/licenses/by-nc/4.0/) which permits copy and redistribute the material just in noncommercial usages, provided the original work is properly cited. 
in the cytokines pathways and also reduce the potential side effects of IFN. Pentoxifylline is a derivative of xanthine is considered a non-selective inhibitor of phosphodiesterase. Pentoxifylline inhibits inflammatory cytokines such as TNF and IL and accelerates immune response deviation from Th1 to Th2 (IL-4, IL-10) cells. So, it can reduce the inflammation caused by immune responses with to inhibit cytokines related to Th1 (IL-5, IL13, and IL-14). Regarding the pentoxifylline's potential, recent studies have investigated the theraputical effects of the drug on patients with MS. For instance, a study in Russia on MS patients with the relapsing-remitting pattern (PRMS) has showed that $34 \%$ of patients who are treated with pentoxifylline and vitamin B supplements do not require steroids in the acute phase of the disease [14]. Thus, it seems that the drug could be used in the treatment of the disease in the prevention phase. Moreover, in another study conducted in Germany, pentoxifylline would reduce the side effects of interferons [15]. However; the therapeutic role of pentoxifylline in patients suffering from MS has not been well understood. The number of studies is limited and their results are subjected to clinical controversies.

\section{Objectives}

In this study, we aim to investigate the effect of pentoxifylline on both the frequency of attacks and expanded disability status scale (EDSS) in MS patients with a relapsing-remitting pattern. To this goal, we conduct a set of analysis on MS patients with 20 - 40 years old, in Kerman city.

\section{Patients and Methods}

Our study was a double-blinded clinical trial with a non-randomized sampling method, in Shafa hospital in Kerman city which was performed during 18 months. All the patients participating in the study filled in a written consent form. Our criteria for entering the study were: 1- new cases of MS (according to the new revised McDonald criteria [1], 2- patients with a relapsing-remitting pattern of disease, 3- ages between 20 - 40 years. Patients with other forms of multiple sclerosis or other diseases (such as autoimmune diseases), any drugs consumption (such as immunomodulatory drugs and acetylsalicylic acid) and pregnant and lactating women, were excluded from the study. The patients were divided into 2 groups according to the type of treatment. Patients in group 1 received IFN- $\beta$ and oral pentoxifylline $800 \mathrm{mg}$ (slow release $400 \mathrm{mg}$ tablets) daily for one month period twice in one year (in the first and seventh month). In previous studies showed that an $800 \mathrm{mg}$ daily dose of pentoxifylline is effective on cytokine production [12] and the therapeutic dose is also safe [4]. Patients in the second group were treated by IFN- $\beta$ and a placebo with the same dose as the inter- vention group. In both treatment groups the standard dose of IFN- $\beta$ was administered and the proper use (needle exchange, cooling, alternating injection sites) was taught to the patients. The number of monthly attacks and also EDSS score were evaluated by clinical examination (by a single physician) at three times: at the beginning of the treatment, 6 months after treatment and in the end of treatment (end of the year). Nowadays EDSS is the most frequently used scale to assess disability in MS patients worldwide and is currently accepted as the standard scale of disability assessment in Iran. EDSS varies from a score of 0 (functional and neurologically normal) to 10 (death due to MS) [1]. All patients were allowed to use ibuprofen, acetaminophen, and ranitidine. All patients were trained to refer to the hospital or call in case of any complications. The participation of all the patients in the study was made possible by an informed written consent and ethic committee of Kerman University of Medical Sciences approved the study. The trial was registered with the Iranian Clinical Trials Registry (IRCT201301148430N7; www.irct.ir).

The averages of the 2 groups were compared by t-test and for nonparametric variables the Mann-Whitney $U$ test was used. For adjusting the confounding factors the Poisson regression was used. A P value below 0.05 was considered as statistically significant.

\section{Results}

Initially, 63 patients with a relapsing-remitting pattern of multiple sclerosis were included in the study. Of the initial population, 18 were excluded from the study due to the lack of desire to continue. One patient died in the fifth month (of causes unrelated to the study). Finally, based on a predetermined sample size, 44 patients were studied. Four of the patients complained of dyspepsia, which during the period of reference, simple gastrointestinal treatments and changes in the diet and eating habits were prescribed that were resolved during the follow-ups and did not have any problems during the rest of the treatment. None of the 2 groups showed any serious adverse effects. Subjects were divided randomly into 2 groups (the controlled and the intervention group), so in each group, 22 (50\%) patients were enrolled. In this study, the average age in the control group was $28.2 \pm 6.1$ years and in the intervention group it was $30.7 \pm 6.7$ years, the average age difference between the patients in the two groups of the study, was not statistically significant (Table 1 ).

Among the 44 patients enrolled, 9 patients were male, 2 males (22.2\%) belonged to the control group, and 7 males $(77.8 \%$ ) belonged to the intervention group. The rest of the 35 enrolled patients remaining were woman, 20 patients $(57.1 \%)$ were in the control group and 15 patients (42.9\%) in the intervention group. There was no statistically significant difference 
in the sex distribution among the study groups (Table 1). The average EDSS score at the time of the patients' initial entry in the study, in the control group was 0.75 \pm 0.85 and in the intervention group it was $1.25 \pm 0.98$, respectively. Tests showed that at the baseline stage in terms of the EDSS there was no statistically significant difference between the intervention and the control group (Table 1 ).

The average EDSS score at the first 6 months was 0.61 \pm 0.75 and $1.13 \pm 0.94$ in the control group and intervention group, respectively. There was no statistically significant difference between the intervention and the control group in terms of EDSS, in the first six months (Table 1). At the end of the one year period, the average EDSS score in the control group was $0.61 \pm 0.67$ and in the intervention group was $1.18 \pm 0.9$, respectively. Analysis showed that there was statistically significant difference between the intervention and the control group in terms of EDSS $(\mathrm{P}=0.039)$ and the average EDSS in intervention group was significantly more than the control group (Table 1). In the attack frequency variable there was no statistically significant difference between the intervention and the control group during the first year of the disease.

Also there was no statistically significant difference in the timing of the attacks (no attack, attack in the first six months and attack in the second six months) between the study groups. In our study the attack frequency in the first group was $0.04 \%$ per year and in the second group no clinical attacks were documented. Over all the multi-variable analysis showed no EDSS score difference during the first year of the disease between the two study groups, thus pentoxifylline did not show any effect in reducing the EDSS score in MS patients, furthermore the attack frequency was not affected by pentoxifylline.

Table 1. Clinical Characteristics of RRMS Patients Treated With IFN and Placebo or Associated With Pentoxifylline

\begin{tabular}{lccc}
\hline Characteristics & IFN & IFN-PTX & P Value \\
\hline Gender, female/male & $20 / 2$ & $15 / 7$ & 0.132 \\
Age, $\mathbf{y}$ & $28.22 \pm 6.06$ & $30.68 \pm 6.7$ & 0.211 \\
EDSS at inclusion & $0.75 \pm 0.85$ & $1.25 \pm 0.98$ & 0.092 \\
EDSS after 6 months & $0.61 \pm 0.75$ & $1.13 \pm 0.94$ & 0.066 \\
EDSS after 12 months & $0.61 \pm 0.67$ & $1.18 \pm 0.9$ & 0.039 \\
\hline
\end{tabular}

\section{Discussion}

In this study, we evaluated the clinical effects of the synergic use of INF-1 $\beta$ with pentoxifylline on the number of attacks and the EDSS score of patients with RRMS during the first year of illness. Although pentoxifylline has shown synergistic effects in in-vivo and in-vitro conditions in RRMS patients, the results of our study, adding the oral pentoxifylline to INF-1 $\beta$ did not improve the clinical conditions of the patients with MS and there was no obvious difference between the EDSS and the number of attacks in the two groups. Treatment with IFN- $\beta$ in multiple sclerosis inhibits the production and migration of the T lymphocytes and also changes the cytokine profile to generate anti-inflammatory cytokines. Pentoxifylline have shown in numerous studies that they inhibit inflammatory factors such as TNF and interleukins and also accelerates the conversion of the immune response from Th1 to Th2 (IL-4, IL-10) cells $[16,17]$.

Effects of simultaneous treatment by pentoxifylline and INF-1 $\beta$ in MS patients, has been studied both in laboratory researches and human cases and this therapeutic synergy has been shown to be an appropriate method to correct cytokine balance in MS patients [15]. Results of our study were supported by the results of the study conducted by Pede et al. in 2005, in which no clear difference in the overall $\mathrm{T}$ cells levels was found between the two treatment groups (which were similar to ours) [17]. In a study by van Oosten et al. [4], no convincing and consistent effects of pentoxifylline were shown on the inflammatory cells and the EDSS of MS patients. This study did not support the therapeutic effect of pentoxifylline in patients with MS. However, the results of our study do not contradict the results found in other studies. Bisaga et al. in a study noted that the use of pentoxifylline and vitamin $B$ reduces the need of steroids in the acute phase [14]. In a study performed by Odinak et al. they reported that a combination of antioxidants and neuroprotective agents such as pentoxifylline is associated with a reduction in the number of relapses and significantly reduces the need for corticosteroids by 2 - 3 times [18].

In another that done by Weber et al. [15], both laboratory data and human evaluations showed that the simultaneous treatment with pentoxifylline and INF-1 $\beta$ is an appropriate method to correct the balance of cytokines in MS patients. In a study by Rieckmann et al. [12], the results showed that pentoxifylline regulates the immune responses in accordance with a response that is similar to the response caused by Th2 cells, in a manner that pentoxifylline decreases IL-12, IFN- $\gamma$ and TNF- $\alpha$ in mononuclear cells of patients with MS, therefore, pentoxifylline can be useful in the treatment of autoimmune diseases that have cellular responses that are similar to the Th1 response.

It seems that the difference found in the results obtained from other studies and our study are due to the differences in matters like: the pattern of MS that was studied, time course disease, the use of simultaneous medical compounds including B vitamins, other antioxidant factors and the therapeutic doses used in different studies. Further studies in larger populations with longer therapeutically durations and for the other forms of MS and the evaluation of immunological parameters associated with MS and the health benefits associated with the immunomodulatory effects of pentoxifylline is required. Also, the finding from this controlled trial may not be generalizable. In conclusion our findings show that add- 
ing pentoxifylline to IFN does not have any synergistic therapeutic effect on relapse frequency and EDSS in patients with relapsing-remitting multiple sclerosis.

\section{Acknowledgements}

Authors would like to thank the research Neurology Research Center for all their support and help, DR. Iranmanesh for his invaluable advice and all MS patients, who kindly participate in this research project (91/168).

\section{Footnote}

Funding/Support:Kerman University of Medical Sciences.

\section{References}

1. Lublin FD, Miller AE. Multiple sclerosis and other inflammatory demyelinating diseases of the central nervous system. In: Bradley WG, Daroff RB, Fenichels GM, editors. Neurology in clinical practice. 5th ed. Philadelphia: Butterworth-Heinemann ; 2008. pp.1583-608.

2. Riley CS, Tullman MJ. Multiple sclerosis. In: Rowland LP, Pedley TA, editors. Merritt's neurology. 12th ed. Philadelphia: Lippincott Williams \& Wilkins; 2010. pp. 904-7.

3. Ropper AH, Samuels MA. Multiple sclerosis and allied demyelinating diseases. In: Ropper AH, Samuels MA, editors. Adams \& Victor's Principles of Neurology. 9th ed. New York: McGraw-Hill Press; 2009. pp. 874-95.

4. van Oosten BW, Rep MH, van Lier RA, Scholten PE, von Blomberg $\mathrm{BM}$, Pflughaupt KW, et al. A pilot study investigating the effects of orally administered pentoxifylline on selected immune variables in patients with multiple sclerosis. J Neuroimmunol. 1996;66(1-2):49-55. [PubMed: 8964913]

5. Olsson T. Cytokines in neuroinflammatory disease: role of myelin autoreactive T cell production of interferon-gamma.J Neuroimmunol.1992;40(2-3):211-8. [PubMed:1430152]

6. Selmaj KW, Raine CS. Tumor necrosis factor mediates myelin and oligodendrocyte damage in vitro. Ann Neurol. 1988;23(4):339-46. doi:10.1002/ana.410230405. [PubMed:3132891]

7. Puccioni-Sohler M, Rieckmann P, Kitze B, Lange P, Albrecht M, Felgenhauer K. A soluble form of tumour necrosis factor receptor in cerebrospinal fluid and serum of HTLV-I-associated myelopathy and other neurological diseases. J Neurol.1995;242(4):239-42. [PubMed: 7798123]

8. Beck J, Rondot P, Catinot L, Falcoff E, Kirchner H, Wietzerbin J. Increased production of interferon gamma and tumor necro- sis factor precedes clinical manifestation in multiple sclerosis: do cytokines trigger off exacerbations? Acta Neurol Scand. 1988;78(4):318-23. [PubMed: 3146861]

9. Wang X, Ma C, Wu J, Zhu J. Roles of T helper 17 cells and interleukin-17 in neuroautoimmune diseases with emphasis on multiple sclerosis and Guillain-Barre syndrome as well as their animal models. J Neurosci Res. 2013;91(7):871-81. doi: 10.1002/jnr.23233. [PubMed: 23653308]

10. Tumani H, Kassubek J, Hijazi M, Lehmensiek V, Unrath A, Sussmuth S, et al. Patterns of TH1/TH2 cytokines predict clinical response in multiple sclerosis patients treated with glatiramer acetate. Eur Neurol. 2011;65(3):164-9. doi: 10.1159/000324035. [PubMed: 21372576]

11. Awad AM, Stuve O. Immunopathogenesis of multiple sclerosis: new insights and therapeutic implications. Continuum (Minneap Minn). 2010;16(5 Multiple Sclerosis):166-80. doi: 10.1212/01. CON.0000389940.92283.aa. [PubMed: 22810604]

12. Rieckmann P, Weber F, Gunther A, Martin S, Bitsch A, Broocks A, et al. Pentoxifylline, a phosphodiesterase inhibitor, induces immune deviation in patients with multiple sclerosis. J Neuroimmunol.1996;64(2):193-200. [PubMed: 8632062]

13. All about multiple sclerosis: MS news and information on diagnosis, symptoms, and treatments. 2011. Available from: http://mult-sclerosis.org/.

14. Bisaga GN, Odinak MM, Boiko AN, Mel'nik Iu B, Popova NF. [Possibilities of treatment of multiple sclerosis exacerbations without corticosteroids: a role of metabolic and antioxidant therapy] Zh Nevrol Psikhiatr Im S S Korsakova. 2011;111(2):44-8. [PubMed: 21350423]

15. Weber F, Polak T, Gunther A, Kubuschok B, Janovskaja J, Bitsch A, et al. Synergistic immunomodulatory effects of interferonbeta1b and the phosphodiesterase inhibitor pentoxifylline in patients with relapsing-remitting multiple sclerosis. Ann Neurol. 1998;44(1):27-34. doi:10.1002/ana.410440109. [PubMed: 9667590]

16. Jafarzadeh A, Ebrahimi HA, Bagherzadeh S, Zarkesh F, Iranmanesh F, Najafzadeh A, et al. Lower serum levels of Th2-related chemokine CCL22 in women patients with multiple sclerosis: a comparison between patients and healthy women. Inflamma tion. 2014;37(2):604-10. doi: 10.1007/s10753-013-9775-z. [PubMed: 24254331]

17. Pede PD, Visintini D, Telera A, Cucurachi L, Campanini C, Immovilli P, et al. Immunomodulatory effects of IFN-beta1a treatment alone or associated with pentoxifylline in patients with relapsing-remitting multiple sclerosis (RRMS). J Interferon Cytokine Res. 2005;25(8):485-9. doi: 10.1089/jir.2005.25.485. [PubMed: 16108731]

18. Odinak MM, Bisaga GN, Zarubina IV. [New approaches to antioxidant therapy in multiple sclerosis]. Zh Nevrol Psikhiatr Im S S Korsakova. 2002;Suppl:72-5. [PubMed:12418396] 